\title{
LE RÉCIT DE ROUSSEAU COMME ÉPREUVE DE LA LIBERTÉ DE CONSCIENCE
}

\author{
André CHARRAK
}

RÉSUMÉ : On sait que, selon Rousseau, la voix de la conscience se prononce dans l'instant et ratifie immédiatement le bien que la raison lui présente. La corruption tenace que fortifie le progrès des connaissances, le tumulte des conversations ou des spectacles empêchent cependant l'homme modeme d'écouter sa conscience, au moment où la société requiert son engagement : l'actualité des rapports corrompus qui enserrent l'individu prend le pas sur la présence en son cœur des impératifs moraux. C'est la raison pour laquelle la philosophie morale de Rousseau ne peut nullement se dispenser d'une théorie de la mémoire : celle-ci permet de dégager le dictamen de la conscience des passions passagères qui nous asservissent aux contemporains; elle dégage l'horizon d'une liberté authentique et d'une responsabilité qui fait toute la permanence de la personne, au regard de l'immortalité de l'âme. En somme, nous montrons ici qu'aux yeux de Rousseau, la réforme s'accomplit dans un récit de conversion.

MOTS-CLES : Bayle, conscience, mémoire, passion, raison, Rêveries du promeneur solitaire, réforme.

ABSTRACT : It is known that, according to Rousseau, the voice of conscience speaks instantaneously, and immediately ratifies the good that reason presents to it. The ferocious corruption that strengthens the progress of knowledge, the tumult of conversations or spectacles, however, prevent modern man from listening to his conscience, at the moment when society requires its engagement: the actuality of corrupted relations that enclose the individual gains ground against the presence of moral imperatives in his heart. Therefore Rousseau's moral philosophy cannot dispense with a theory of memory: the latter permits the dictamen of the conscience to be disengaged from the transitory passions that subject us to contemporaries; it disengages the horizon of an authentic freedom and a responsibility that creates the person's permanence, in regard to the soul's immortality. In sum, we show here that in Rousseau's eyes, reform is accomplished in a conversion narrative.

KEYWORDS : Bayle, conscience, memory, passion, reason, Rêveries du promeneur solitaire, reform.

Revue de synthèse : $4^{e}$ S. Nos 3-4, juil.-déc. 1996, p. 425-439. 
ZusammenFassung : Nach Rousseau spricht die Stimme des Gewissens im Augenblick ihr Urteil aus und bestätigt unmittelbar das ihm von der Vernunft vorgehaltene Gute. Die zähe, mit dem Fortschritt der Wissenschaften erstarkende Verdorbenheit, der Rausch der Gespräche und Schauspiele hindern jedoch den modernen Menschen daran, auf sein Gewissen in eben dem Augenblick zu hören, wo er von der Gesellschaft zum Einsatz seiner Person aufgefordert wird: die aktuelle Verstrickung des Individuums in verdorbenen Verhältnissen überwiegt die Gegenwart der sittlichen Imperative in seinem Herzen. Daher ist bei der Moralphilosophie Rousseaus eine Theorie des Gedächtnisses unverzichtbar : diese leistet tatsächlich die Freilegung des dictamen des Gewissens von den flüchtigen Leidenschaften, die uns zu Sklaven unserer Zeitgenossen machen; sie erschließt den Horizont der wahren Freiheit und der Verantwortlichkeit, welche den ganzen Bestand der Person hinsichtlich der Unsterblichkeit der Seele ausmacht. Mit einem Wort: wir wollen hier zeigen, daß sich in Rousseaus Augen die Reform in einem Bekenntnisbericht vollzieht.

STICHWÖTER : Bayle, Gewissen, Gedächtnis, Leidenschaft, Vernunft, Rêveries du promeneur solitaire, Reformation.

André Charrak, ancien élève de l'École normale supérieure de Fontenay/Saint-Cloud, agrégé de philosophie, est AMN à l'université de Caen. Il réalise une thèse sur le problème de la résonance du corps sonore au XVIII siècle.

Adresse : Université de Caen, UFR des sciences de l'homme, Esplanade de la Paix, 14052 Caen Cedex. 
Au livre IV de l'Émile, Rousseau affirme que « l'étude convenable de l'homme est celle de ses rapports ${ }^{1}$. Ces rapports définissent d'abord la relation de l'individu physique aux choses matérielles, et cette relation découvre au petit homme une nécessité aveugle, rebelle à ses caprices. L'enfant apprend à vouloir ce qu'il peut, et assoit de la sorte l'autonomie à laquelle il est destiné, en tant qu'être libre : à cet égard, l'île de Robinson Crusoé, «c'est le vrai château-en-Espagne de cet heureux âge, où l'on ne connaît d'autre bonheur que le nécessaire et la liberté ${ }^{2}$. En revanche, pour un homme fait, qui vit à l'époque de Rousseau, ces rapports, qui se tissent désormais avec les autres hommes, sont déterminés par l'opinion et fortifiés par l'imagination. L'homme moderne épuise toutes ses forces dans la satisfaction d'un amour propre qui consiste, selon le second Discours, en « un sentiment relatif par lequel on se compare, qui demande des préférences, dont la jouissance est purement négative, et qui ne cherche plus à se satisfaire par notre propre bien, mais seulement par le mal d'autrui ${ }^{3}$. Tel est précisément l'objet des Confessions, que de fournir à la postérité un nouveau point de comparaison qui restitue une image authentique de l'homme ( « un homme dans toute la vérité de la nature " ${ }^{4}$, écrit Rousseau), à la place des opinions variées et dégradées : l'ouvrage peut ainsi «servir de première pièce de comparaison pour l'étude des hommes, qui certainement est encore à commencer $»^{5}$. Le problème est que l'auteur lui-même doit intégrer au récit de ses Confessions la description des rapports sociaux dans lesquels il fut engagé, parce qu'il n'eut pas le bonheur d'être placé chez un artisan. Ce procédé répond à une exigence que Rousseau formula dès 1745, dans un texte intitulé Idée de la méthode dans la composition d'un livre:

«Dans les recherches morales [...] je commencerai par examiner le peu que nous connaissons de l'esprit humain pris en lui-même et considéré comme individu, j'en tirerai en tâtonnant quelques connaissances obscures et incer-

1. Émile, liv. IV, in Jean-Jacques Rousseau, CEuvres complètes, 5 vol., Paris, Gallimard ("Bibliothèque de la Pléiade »), 1959-1995 (cité par la suite comme $O C$ suivi du numéro du volume en chiffres romains), IV, p. 831. Sur cet aspect de l'anthropologie rousseauiste, voir Robert DeratHÉ, «L'homme selon Rousseau », in Etudes sur le Contrat social de J.-J. Rousseau, Paris, Les Belles Lettres, 1964, reprod. in Pensée de Rousseau, Paris, Seuil (« Points », 168), 1984.

2. Émile, liv. III, in $O C$, IV, p. 455.

3. Discours sur l'origine et les fondements de l'inégalité parmi les hommes, I, in OC, III, p. 669 .

4. Confessions, liv. I, in $O C, \mathrm{I}, \mathrm{p} .5$.

5. Confessions, Préambule, in $O C$, I, p. 3. 
taines, mais abandonnant bientôt ce ténébreux labyrinthe, je me hâterai d'examiner l'homme par ses relations, et c'est de là que je tirerai une foule de vérités lumineuses $[. .$.$] qui recevraient encore du jour par comparaison { }^{6}$.

On trouve l'expression d'une exigence analogue au livre VIII des Confessions: « Pour me bien connaître il faut me connaître dans tous mes rapports bons ou mauvais. Mes confessions sont nécessairement liées avec celles de beaucoup de gens ${ }^{7}$. Une fonction possible du récit autobiographique serait ainsi de manifester la permanence d'une humanité authentique, au sein de rapports corrompus qui, proprement, aliènent l'individu $^{8}$. D'où l'hypothèse que nous nous proposons d'examiner ici : l'autobiographie doit attester qu'une conscience destinée à l'exercice moral de la liberté se maintient, tandis que la personne se trouve premièrement captée par les rapports sociaux dans lesquels elle s'inscrit. Précisons. On considère avec justesse que l'enjeu des Confessions, pour Rousseau, est de s'avouer à la postérité, de paraître tel qu'il est, afin de devenir vraiment ce qu'il est destiné à être, selon le principe que "quiconque a le courage de paraitre toujours ce qu'il est deviendra tôt ou tard ce qu'il doit être ». Mais il est intéressant de considérer que cette épreuve de l'aveu sanctionne un travail intérieur, qui se décrit assez bien comme un examen de conscience. Les écrits autobiographiques de Rousseau doivent ainsi être également examinés dans la logique de leur constitution, c'est-à-dire dans le travail de mémoire qu'ils requièrent. De ce point de vue, il apparaît que cet exercice peut devenir l'adjuvant nécessaire de la voix de la conscience, du moins pour l'homme moderne, dont nous verrons qu'il ne peut immédiatement l'écouter. Nous voulons montrer comment la réforme rousseauiste implique un certain travail de la mémoire, qui intervient dans l'émergence de la personne morale - au moins au titre de condition matérielle, et pour ce qui concerne l'homme moderne.

Tout d'abord, il est important de rappeler que, dans la société française de son siècle, Rousseau considère que la voix de la conscience est très souvent étouffée. Certes, il affirme dans la Quatrième promenade que toute sa vie, il mit un terme aux longues délibérations en consultant sa conscience : «Dans toutes les questions de morale difficiles [...], je me suis toujours bien trouvé de les résoudre par le dictamen de la conscience plutôt que par les lumières de la raison " ${ }^{9}$. Mais précisément, s'il est question

6. $O C$, II, p. 1245.

7. Confessions, liv. VIII, in $O C, \mathrm{I}, \mathrm{p.} 400$.

8. Émile, liv. IV, in $O C$, IV, p. 515 .

9. Les Rêveries du promeneur solitaire, Quatrième promenade, in $O C, \mathrm{I}, \mathrm{p} .1028$. 
d'un dictamen, c'est que «l'homme de l'homme » doit vaincre ses propres résistances afin d'écouter en lui le «verbe intérieur" qui tranche infailliblement en matière morale. Les lumières de la raison, outre qu'elles portent souvent sur des objets trop généraux, se confondent volontiers avec les sophismes qu'inspire notre intérêt. Quand l'homme ne recourt pas aux « subtilités du raisonnement », c'est-à-dire, lorsqu'il ne justifie pas des actions simplement intéressées par des raisons plus générales qui pourraient indifféremment être remplacées par des raisons opposées, selon les circonstances - alors il se rend compte que la conscience, hic et nunc, en face de tel problème moral, doit emporter une décision sans équivoque : « le meilleur de tous les casuistes est la conscience, et ce n'est que quand on marchande avec elle qu'on a recours aux subtilités du raisonnement ${ }^{10}$. Le problème est que la conscience ne peut faire entendre sa voix lorsque nous sommes portés hors de nous-mêmes, par l'amour-propre qui rapporte nos choix, non au dictamen de la conscience, mais à l'approbation superficielle des autres hommes dont nous voulons être appréciés : «Ici-bas mille passions ardentes absorbent le sentiment interne et donnent le change aux remords. Les humiliations, les disgrâces qu'attire l'exercice des vertus empêchent d'en sentir tous les charmes " ". Il n'est pas interdit de songer ici à Bayle, dont Rousseau inverse l'argumentation sur les rapports entre conscience et société. L'existence d'une conscience morale, si elle est incapable, selon Bayle, d'infléchir directement notre conduite qui demeure naturellement mauvaise, détermine cependant les jugements que nous portons sur la conduite d'autrui. Par suite, il peut bien nous arriver de respecter les règles de la morale, afin d'obtenir l'approbation d'autrui que recherche notre amour-propre ${ }^{12}$. Rousseau accorde bien que nous nous conformons volontiers aux maximes admises dans la société où nous évoluons; mais précisément, ces maximes sont mauvaises, et ne sanctionnent pas les décrets de la conscience. Bien au contraire, c'est la conscience qui se trouve travestie par les préjugés du public. Depuis la Profession de foi $d u$ vicaire savoyard, nous savons que « c'est du système moral formé par ce double rapport à soi-même et à ses semblables que naît l'impulsion de la conscience ${ }^{13}$ : mais aujourd'hui, comme la société historique est mauvaise, il y a conflit entre les rapports de dissimulation que nous entretenons avec les autres membres de la multitude, et l'authentique rapport à soi qui est la condition de l'écoute de la conscience. L'impulsion de la conscience est maintenant filtrée par les opinions qui règlent la sociabilité contempo-

10. Émile, liv. IV, in $O C$, IV, p. 594.

11. Émile, liv. IV, in $O C$, IV, p. 591.

12. Sur cette question, voir Élisabeth Labrousse, «Obscurantisme et Lumières chez Pierre Bayle », in Studies on Voltaire, t. 26, 1963, repris in Notes sur Bayle, Paris, Vrin, 1987.

13. Emile, liv. IV, in $O C$, IV, p. 600. 
raine, et cette déformation se marque à ce que la conscience est confondue avec les préjugés: désormais, "la conscience est timide, elle aime la retraite et la paix; le monde et le bruit l'épouvantent, les préjugés dont on la fait naître sont ses plus cruels ennemis, elle fuit ou se tait devant eux ${ }^{14}$. On aurait tort de considérer que Rousseau ait estimé que l'expression de sa propre conscience ne fût pas affectée par la dégénérescence du système moral : il en fut bien la victime, pour trois raisons dont les récits autobiographiques fournissent plusieurs illustrations. Tout d'abord, on sait que chez Rousseau la conscience est amour spontané d'un bien qu'elle ignore, et que la raison doit lui présenter ce bien - on ne peut même parler de moralité (voir la Lettre à Christophe de Beaumont) que si une connaissance rationnelle éclaire les premiers mouvements de notre conscience. Comme l'écrit R. Derathé, « il y a bien de la différence entre [une] impulsion primitive et la conscience complète, développée et devenue active par l'usage de la raison $"{ }^{15}$. Et la raison, lorsqu'au moins elle n'est pas corrompue par les maximes des philosophes, découvre un ordre social dans lequel le mieux que l'on puisse faire (pour Rousseau, abandonner ses enfants) heurte encore les mouvements naturels de l'âme, qui protestent chez Thérèse : « [...] je ne mis aucun mystère à ma conduite, non seulement parce que je n'ai jamais rien su cacher à mes amis, mais parce qu'en effet je n'y voyais aucun mal. Tout pesé, je choisis pour mes enfants le mieux ou ce que je crus l'être ${ }^{16}$ - la moralité de l'acte est suspendue au bien que la faculté rationnelle est susceptible de présenter. Une seconde raison tient bien entendu aux passions, dont Rousseau souligne qu'il en fut longtemps la victime, et qui assourdissent la voix de la conscience. Mais on peut envisager l'efficacité redoutable des passions d'un autre point de vue, qui correspond au troisième facteur d'aliénation. Avançons cette thèse : ce qui caractérise la prégnance des rapports sociaux, leur capacité à capter l'individu hors de lui-même (ainsi dans les passions), c'est, pourrions-nous dire, leur actualité. Dans la Quatrième promenade, Rousseau souligne que les rares mensonges qu'il doit se reprocher (ainsi d'avoir affirmé en public qu'il n'eut point d'enfants) furent tous prononcés alors qu'il était présent, non à lui-même, mais à des individus qui dissimulaient leurs véritables intentions. Lorsque la personne est captée hors d'elle-même, c'est que le sentiment actuel de son existence se confond avec son inscription dans l'ordre social du paraître - l'individu ne peut alors écouter la voix de la conscience, qui lui commande de ne point mentir:

14. Émile, liv. IV, in $O C$, IV, p. 601

15. R. Derathe, Le Rationalisme de J.-J. Rousseau, Paris, Presses universitaires de France, 1948, p. 110.

16. Confessions, liv. VIII, in $O C, \mathrm{I}, \mathrm{p} .358$. 
« C'est encore par cette première et irrésistible impulsion du tempérament [il s'agit de la mauvaise honte, qui déjà lui faisait accuser Marion] que dans des moments imprévus et rapides la honte et la timidité m'arrachent souvent des mensonges auxquels ma volonté n'a point de part, mais qui la précèdent en quelque sorte par la nécessité de répondre à l'instant ${ }^{17}$.

L'actualité des rapports sociaux se substitue à l'authentique présence à soi, en quoi réside la véritable autonomie. Dans ce cas, la volonté n'est pas véritablement engagée dans la décision, que détermine mécaniquement la configuration particulière du groupe - nous reviendrons plus tard sur ce point. Cette situation, où l'actualité du paraître prend le pas sur la présence à soi, contamine aussi bien la raison, dans la mesure où celle-ci doit recourir aux signes d'institution dont la principale fonction est de dissimuler ce que l'on pense vraiment :

« De tout ce qu'on dit, de tout ce qu'on fait, de tout ce qui se passe en ma présence, je ne sens, je ne pénètre rien. Le signe extérieur est tout ce qui me frappe. Mais ensuite tout cela me revient : je me rappelle le lieu, le temps, le ton, le regard, le geste, la circonstance, rien ne m'échappe. Alors sur ce qu'on a fait ou dit je trouve ce qu'on a pensé, et il est rare que je me trompe " 18 .

La mémoire aurait ainsi pour fonction de mettre l'individu à l'écart d'une situation dont il ne peut, lorsqu'il s'y trouve actuellement engagé, démêler la duplicité. Reste à établir que de cette façon, la personne morale se trouve effectivement restaurée.

Auparavant, il faut bien rappeler que l'individu peut se libérer des rapports sociaux aliénants, s'il choisit précisément de se réformer. Le premier mouvement de la réforme doit être de restituer à la voix de la conscience toute sa clarté, c'est-à-dire qu'il faut rentrer en soi afin de l'entendre, puisqu'elle ne s'est jamais tue. À cette fin, il convient de réduire l'amourpropre, c'est-à-dire de rejeter les maximes qui nous valent l'estime des contemporains auxquels nous n'étions reliés que par des rapports factices de comparaison : « $[\ldots]$ je ne songeai plus qu'à $[\ldots]$ rendre [ma réforme] solide et durable, en travaillant à déraciner de mon cœur tout ce qui tenait encore au jugement des hommes, tout ce qui pouvait me détourner par la crainte du blâme de ce qui était bon et raisonnable en soi » ${ }^{19}$. Il s'agit, dans

17. Rêveries, Quatrième promenade, in $O C, \mathrm{I}, \mathrm{p} .1033$.

18. Confessions, liv. III, in $O C, \mathrm{I}$, p. 115.

19. Confessions, liv. VIII, in $O C, \mathrm{I}, \mathrm{p} .364$. 
la réforme, de réduire tout à fait la part de nos actions qui est déterminée de l'extérieur, par l'autorité. Cela ne signifie pas qu'une liberté totalement indéterminée sera désormais en charge de notre moralité ; bien plutôt, nous devons tenir que la raison admettra librement ce qu'elle peut comprendre des dogmes historiquement formulés, cependant que la conscience se prononce sur les vérités incompréhensibles. La réforme individuelle, de même que le protestantisme authentique que Rousseau appelle de ses vœux, réside avant tout dans le libre examen. C'est ce que les Lettres écrites de la Montagne rappellent aux pasteurs de Genève, et $\mathrm{H}$. Gouhier signale que, sur ce point, le citoyen de Genève enregistre un caractère notable du calvinisme de son temps: "le libre examen devient explicitement et presque officiellement principe premier de la religion chrétienne ${ }^{20}$. Pour ce qui concerne, chez Rousseau, les rapports de l'individu à la société moderne dans laquelle il est engagé, on doit admettre que la réforme impose parfois un refus de cet engagement. En effet, si ses actions sont toujours reprises dans un ordre social perverti, il doit choisir de ne plus agir, plutôt que d'être coupable d'entretenir un système moral inadéquat. Le premier résultat positif de cette réforme (on ne peut que songer à la «belle âme » dont parlera Hegel) est de nous décharger du fardeau de la culpabilité. Fénelon, que Rousseau dit avoir beaucoup fréquenté, écrivait déjà qu'un « homme n'est coupable que quand il fait ce qu'il peut s'empêcher de faire ${ }^{21}$. Rousseau adopte tout à fait ce point de vue, et déclare à $M$. de Malesherbes : « [...] on n'est jamais excusable de faire mal ce qu'on fait volontairement ${ }^{22}$. Mais l'âme de Rousseau ne s'offre pas une bonne conscience aussi facilement, et le repli sur soi, la volonté de se circonscrire, est investie d'une double signification. D'abord, Rousseau prend soin de présenter son attitude comme un fait exceptionnel, que lui impose l'état définitivement mauvais des rapports qu'il entretient avec ses contemporains :

« Il ne serait pas même bon dans la présente constitution des choses, qu'avides de ces douces extases ils s'y dégoûtassent de la vie active dont leurs besoins toujours renaissants leur prescrivent le devoir. Mais un infortuné qu'on a retranché de la société humaine et qui ne peut plus rien faire ici bas d'utile et de bon pour autrui ni pour soi, peut trouver dans cet état à toutes les félicités des dédommagements que la fortune et les hommes ne lui sauraient ôter ${ }^{23}$.

20. Henri GounIER, Les Méditations métaphysiques de J.-J. Rousseau, Paris, Vrin, 1984 , p. 229.

21. Fénelon, Lettres sur divers sujets de métaphysique et de religion, lettre I, chap. III, "Sur le culte de Dieu, l'immortalité de l'âme et le libre arbitre ", Paris, Firmin Didot frères, 1861, p. 138.

22. Quatre lettres à M. le Président de Malesherbes contenant le vrai tableau de mon caractère et les vrais motifs de ma conduite, lettre IV, in $O C, \mathrm{I}, \mathrm{p} .1144$.

23. Rêveries, Cinquième promenade, in $O C, \mathrm{I}, \mathrm{p} .1047$. 
Ensuite, il faut bien comprendre que le jugement de Hegel ( Le cœur [est] la singularité de la conscience, singularité voulant être immédiatement universelle $\gg^{24}$ ) ne concerne, pourrait-on dire, que la première partie de la démarche rousseauiste - certes, il s'agit bien, pour Rousseau, de réaliser l'universel humain : mais cette démarche n'a de sens que pour les autres hommes, en ce qu'ils se rapportent à Rousseau comme à une pièce de comparaison. Aux philosophes qui lui reprochent sa «trahison », c'est-àdire son retrait critique à l'égard de l'entreprise encyclopédique, Rousseau répond que «c'est quelque chose que de donner l'exemple aux hommes de la vie qu'ils devraient tous mener ${ }^{25}$ : l'importance des écrits autobiographiques dans l'ensemble du système de Rousseau s'avère donc capitale. Quoi qu'il en soit, il demeure très significatif que l'expression fondamentale de la liberté vécue par Rousseau consiste dans une négation, opérée semel in vita, à la manière de Descartes - mais la portée de cette négation n'est pas directement gnoséologique, elle est avant tout morale, et vise à circonscrire le domaine propre où la personne découvre son autonomie : " [...] l'espèce de bonheur qu'il me faut, n'est pas tant de faire ce que je veux, que de ne pas faire ce que je ne veux pas ${ }^{26}$. Par conséquent, il s'agit moins de refuser notre assentiment aux sources de connaissance qui nous ont déjà trompés, que de suspendre notre participation à un ordre social qui condamne l'action droite. Il faut éviter de tomber dans une duplicité (notre intention bonne, les malheurs qui la sanctionnent dans le monde), qui affecte notre intégrité : «J'étais actif parce que j'étais fou » ${ }^{27}$. Néanmoins, nous sommes ici confrontés à une réelle difficulté : lorsqu'il entend maintenir l'intégrité de sa personne morale, en face d'un ordre social implacable et corrompu, Rousseau considère que le retrait est seul possible, qui permet à l'âme de se circonscrire. Alors, elle éprouve sa libre disposition à faire le bien. Aussi bien, devons-nous prendre au sérieux telle déclaration de Rousseau : « La vie active n'a rien qui me tente, je consentirais cent fois plutôt à ne jamais rien faire qu'à faire quelque chose malgré moi; et $j$ 'ai cent fois pensé que je n'aurais pas vécu trop malheureux à la Bastille, n'y étant tenu à rien du tout qu'à rester là ${ }^{28}$. Mais dans ce cas, comment peut-on affirmer que l'individu Rousseau demeura effectivement libre, c'est-à-dire librement disposé à faire le bien, tandis que les hommes menacèrent toujours de le corrompre, c'est-à-dire de le faire mentir? Autrement dit,

24. Georg Wilhelm Friedrich Hegel, Phénoménologie de l'esprit, trad. de Jean Hyppoltte, Paris, Aubier, 1941, t. I, p. 309.

25. Quatre lettres à M. le Président de Malesherbes, lettre IV, in $O C, \mathrm{I}, \mathrm{p} .1143$.

26. Ibid., lettre I, in $O C$, I, p. 1132.

27. Ibid., lettre II, in $O C$, I, p. 1134.

28. Ibid., lettre I, in $O C, \mathrm{I}, \mathrm{p} .1132$. 
qu'est-ce qui atteste la permanence de la personne morale, au travers des étapes de la biographie?

III

C'est à ce niveau de l'interrogation que nous découvrons l'importance capitale de la mémoire et, de façon dérivée, celle de l'entreprise autobiographique pour la constitution de la personne morale. Nous l'avons dit, Rousseau n'a menti que lorsque l'actualité de l'amour-propre s'est imposée à lui, en ce qu'elle le contraignait à maintenir immédiatement une apparence au sein des rapports sociaux. Mais dès le moment que la décision d'une réforme est engagée, l'individu se circonscrit à l'écart des fictions tissées par l'amour-propre. Et la conscience peut alors se prononcer sereinement parce qu'elle revient sur la passion, le mensonge, pour ne considérer en eux que ce qui intéresse la personne morale, c'est-à-dire le bien qu'elle aurait dû garder en vue - c'est précisément la fonction de la mémoire, que d'attester qu'il s'agissait déjà du bien et du mal moral, au lieu que l'individu ne percevait que le complexe de ses passions et des préjugés auxquels elles l'inféodaient :

«À mesure que j'avance vers le terme de ma carrière, je sens affaiblir tous les mouvements qui m'ont soumis si longtemps à l'empire des passions. [...] Dans cet état il est naturel que j'aime à tourner les yeux sur le passé duquel je tiens désormais tout mon être, c'est alors que mes erreurs se corrigent et que le bien et le mal se font sentir à moi sans mélange et sans préjugés $»^{29}$.

Il faut donc considérer que les fautes commises dans le passé ne condamnent pas l'homme irrémédiablement, parce que la mémoire permet d'authentifier la présence constante d'un sentiment du bien, émis par la conscience, qui simplement s'est trouvé voilé par nos passions : «Jamais l'instinct moral ne m'a trompé : il a gardé jusqu'ici sa pureté dans mon cœur assez pour que je puisse m'y confier, et s'il se tait quelquefois devant mes passions dans ma conduite, il reprend bien son empire sur elle dans mes souvenirs $"{ }^{30}$. On pourrait presque dire que la mémoire constitue, dans la philosophie religieuse de Rousseau, le fondement anthropologique du pélagianisme: la mémoire permet en effet au méchant de ressaisir l'essence authentique de l'homme, telle qu'elle demeure toujours intacte dans son propre cour - entendons sa destination originelle à faire le bien. Cette perspective permet à Rousseau de montrer comment la liberté peut être restituée, au travers des pires égarements : lorsque j'inscris mon action

29. Lettres morales, lettre IV, in $O C$, IV, p. 1103.

30. Rêveries, Quatrième promenade, in $O C, \mathrm{I}, \mathrm{p} .1028$. 
dans l'ordre des valeurs instituées par l'homme de l'homme, qui donne tout à l'argent, aux mots, au spectacle, alors j'aliène ma liberté dans la dépendance de choses que n'exige pas la conscience; au contraire, lorsque je réalise la réforme en moi-même, la voix de la conscience peut sanctionner, dans les états passionnels révolus, l'absence d'une volonté droite, c'est-àdire affranchie des mobiles simplement sensuels et éphémères. Le remords, c'est l'exercice de mémoire par lequel la personne morale récupère enfin sa liberté : «Quand je me livre aux tentations j'agis selon l'impulsion des objets externes. Quand je me reproche cette faiblesse je n'écoute que ma volonté; je suis esclave par mes vices et libre par mes remords $\gg^{31}$. Encore faut-il préciser, avec le père $A$. Ravier, que ce sentiment est dépourvu, chez Rousseau, de ses déterminations proprement chrétiennes : humilité, appel à la miséricorde et appel à l'aide de Dieu ${ }^{32}$. Corrélativement, il apparaît que la mémoire est seule susceptible de fournir un mobile empirique acceptable, qui justifie l'action droite, malgré les désagréments que celle-ci entraîne au sein des rapports sociaux tels qu'actuellement organisés. C'est au nom d'un eudémonisme du vertueux que Rousseau considère qu'à tout prendre, c'est-à-dire si l'on tient compte de la survie de l'âme, il est préférable d'agir conformément à la voix de la conscience - faute de quoi celle-ci nous reprochera, dans nos souvenirs, tous les manquements dont nous sommes responsables:

«Le souvenir de mes actions bonnes ou mauvaises me fait un bien être ou [un] mal être durable plus réel que celui qui en fut l'objet; ainsi les plaisirs d'un moment m'ont souvent préparé de longs repentirs; ainsi les sacrifices faits à l'honnêteté et à la justice me dédommagent tous les jours de ce qu'ils m'ont une fois coûté et pour de courtes privations me donnent d'éternelles jouissances ${ }^{33}$.

On sait bien qu'aux yeux de Rousseau, l'idée du bonheur comporte celle de la permanence d'un état parfaitement stable, qui paraît incompatible avec le régime chaotique des passions. L'homme moderne ne connaît un bonheur authentique qu'en deux situations, d'ailleurs incommensurables; or il est très significatif qu'en ces deux situations la mémoire remplit une fonction notable ${ }^{34}$. La première réalisation du bonheur réside dans la rêve-

31. Émile, liv. IV, in $O C, \mathrm{IV}$, p. 586.

32. André RAviER, «J.-J. Rousseau et l'éducation d'une conscience d'homme», in J.-J. Rousseau et la crise contemporaine de la conscience, Paris, Beauchesne, 1980.

33. Lettres morales, lettre IV, in $O C$, IV, p. 1103.

34. Nous laissons ici de côté l'usage épicurien du souvenir que Rousseau préconise afin de nous distraire des souffrances actuelles, cf. Quatre lettres à $M$. le Président de Malesherbes, lettre III, in $O C, I$, p. 1139 : " Quand mes douleurs me font tristement mesurer la longueur des nuits, et que l'agitation de la fièvre m'empêche de goûter un seul instant de sommeil, souvent je me distrais de mon état présent, en songeant aux divers événements de ma vie, et les repen- 
rie, dont nous ne présenterons pas ici une exposition maladroite. Rappelons simplement que la perdurabilité de cet état de pure existence constitue une garantie de l'autosuffisance du rêveur : « De quoi jouit-on dans une pareille situation? De rien d'extérieur à soi, de rien sinon de soi-même et de sa propre existence, tant que cet état dure on se suffit à soi-même comme Dieu " ${ }^{35}$. Or la mémoire intervient à deux niveaux dans la constitution de la rêverie : tout d'abord, elle fournit au rêveur un matériau, des souvenirs de personnes et de lieux qu'il peut librement combiner, afin de rendre son extase plus douce encore; ensuite (et c'est, à nos yeux, le plus important), la mémoire peut restituer les rêveries passées et, de cette façon, les approfondir. La remémoration confère aux extases réactivées une densité existentielle supérieure ${ }^{36}:$ "Leurs objets échappaient souvent à mes sens dans mes extases, et maintenant plus ma rêverie est profonde plus elle me les peint vivement. Je suis souvent plus au milieu d'eux et plus agréablement que quand j'y étais réellement ${ }^{37}$ - nous sommes tentés de dire que, par la remémoration, on jouit davantage de soi-même, on se rend davantage semblable à Dieu. Cette suggestion nous fait rejoindre le jugement que Rousseau porte rétrospectivement sur le texte des Confessions. Cet exercice de mémoire écrite, dans lequel l'auteur a soumis tous ses états passés à un jugement orienté par le dictamen de la conscience, n'est pas moins sévère que le regard que Dieu portera sur lui : « [...] je me sentais plutôt porté à mentir dans le sens contraire en m'accusant avec trop de sévérité qu'en m'excusant avec trop d'indulgence, et ma conscience m'assure qu'un jour je serai jugé moins sévèrement que je ne me suis jugé moimême ${ }^{38}$. On rejoint dès lors la vraie réalisation du bonheur, qui consiste dans la jouissance d'une âme immortelle qui se souvient du bien qu'elle aura fait sur la terre ${ }^{39}$. Et dans la Profession de foi du vicaire savoyard, la mémoire du bien réalisé et du mal commis sont tout le contenu déterminé que nous pouvons donner à l'idée d'une survie de l'âme, après la mort :

«ce que je sais bien c'est que l'identité du moi ne se prolonge que par la mémoire, et que pour avoir été le même en effet, il faut que je me souvienne d'avoir été. Or je ne saurais me rappeler après ma mort ce que j'ai été durant ma vie que je ne me rappelle aussi ce que j'ai senti, par conséquent ce que j'ai

tirs, les doux souvenirs, les regrets, l'attendrissement se partagent le soin de me faire oublier quelques moments mes souffrances. "

35. Rêveries, Cinquième promenade, in $O C, \mathrm{I}, \mathrm{p} .1047$.

36. Le rôle du temps dans la rêverie est clairement exposé par Guy BESSE, J.-J. Rousseau, l'apprentissage de l'humanité, Paris, Éditions sociales, 1988, p. 429-430.

37. Rêveries, Cinquième promenade, in $O C$, I, p. 1049.

38. Rêveries, Quatrième promenade, in $O C, \mathrm{I}, \mathrm{p} .1035$.

39. Cet élément est capital dans la théodicée rousseauiste : voir, par ex., Kiyotaka KawaI, "Sur la théodicée du Vicaire savoyard ", in Rousseau, l'Émile et la Révolution, Actes du colloque de Montmorency, 1989, Paris, Universitas, 1992. 
fait, et je ne doute point que ce souvenir ne fasse un jour la félicité des bons et le tourment des méchants ${ }^{40}$.

L'exercice de la mémoire, s'il est orienté par la voix de la conscience, c'est-à-dire s'il résulte d'une réforme, permet à l'homme moderne, dont les actions vertueuses sont actuellement condamnées par la société, de rendre équivalents la connaissance de soi et le regard de Dieu sur lui.

En fonction de cette analyse, que convient-il de penser, pour conclure, de ce que Rousseau appelle souvent sa « destinée »? Soulignons une fois encore l'importance de la notion de rapport, qui doit dissiper la fiction du complot. La réforme entreprise par Rousseau, dans la mesure où elle donne l'exemple d'une humanité authentique, ne peut que provoquer la haine de ceux qui participent à l'ordre social : « ils m'auraient pardonné peut-être de briller dans l'art d'écrire; mais ils ne purent me pardonner de donner par ma conduite un exemple qui semblait les importuner ${ }^{41}$. Mais il faut, une fois encore, faire taire l'amour-propre, qui me met à la portée de ces attaques - Rousseau doit apprendre à ne considérer en elles que la réaction d'une société qui, de toute nécessité, se défend contre celui qui entre en réforme :

« Alors je commençai à me voir seul sur la terre et je compris que mes contemporains n'étaient par rapport à moi que des êtres mécaniques qui n'agissaient que par impulsion et dont je ne pouvais calculer l'action que par les lois du mouvement. Quelque intention, quelque passion que j'eusse pu supposer dans leurs âmes, elles n'auraient jamais expliqué leur conduite à mon égard d'une façon que je pusse entendre. C'est ainsi que leurs dispositions intérieures cessèrent d'être quelque chose pour moi. Je ne vis en eux que des masses différemment mues, dépourvues à mon égard de toute moralité ${ }^{42}$.

De la même façon que l'examen de conscience manifeste au réformé l'asservissement où le tenaient ses passions, de la même façon, il révèle dans l'adhésion des contemporains à l'ordre social une absence totale de volonté - ce sont les groupes auxquels les individus se rapportent qui attaquent mécaniquement Rousseau, et non ces individus qui ne sont jamais présents à eux-mêmes : entendons, qui ne sont jamais libres. Les persécuteurs ne se constituent pas en personnes morales. La comparaison avec des masses différemment mues n'est pas indifférente : n'oublions pas que, selon Rousseau, le fait que je puisse mouvoir mon corps atteste évidemment la réalité de la volonté comme principe actif ${ }^{43}$. Affirmer que les persé-

\footnotetext{
40. Émile, liv. IV, in $O C$, IV, p. 590-591.

41. Confessions, liv. VIII, in $O C, \mathrm{I}, \mathrm{p} .362$.

42. Rêveries, Huitième promenade, in $O C, \mathrm{I}, \mathrm{p} .1078$.

43. FÉnELon écrivait déjà, in op. cit. supra n. 21 , lettre I, chap. I : " J'ai beau vouloir que les autres corps se remuent, il ne s'en meut aucun [...] : mais, pour la masse de mon corps, la
} 
cuteurs réagissent en revanche comme les masses de la mécanique, c'est refuser de lire dans la persécution le produit d'une quelconque spontanéité, d'une liberté ou d'une intention véritable. Nous avons justifié brièvement la référence à Fénelon, mais elle paraît vraiment s'imposer : « $\mathrm{Si}$ une nécessité inévitable et invincible nous fait vouloir tout ce que nous voulons, notre volonté n'est pas plus responsable de son vouloir qu'un ressort de machine n'est responsable du mouvement qui lui est inévitablement et invinciblement imprimé ${ }^{44}$ - telle est bien la situation des dogmatiques et des sceptiques qui assaillent le réformé. C'est l'imagination qui ajoute aux maux réels que supporte cet homme la fiction d'une intention de nuire, qui organiserait toutes les difficultés qu'il rencontre. Puisqu'une raison éclairée nous présente des rapports sociaux où il n'est point question de liberté, mais d'aliénation de soi, alors il faudrait que le réformé se tienne pour simplement soumis aux persécutions, mais non véritablement possédé par celles-ci :

" [...] tout ce que j'avais à faire encore sur la terre étant de m'y regarder comme un être purement passif je ne devais point user à résister inutilement à ma destinée la force qui me restait pour la supporter. Voilà ce que je me disais, ma raison, mon cœur y acquiesçaient et néanmoins je sentais ce cœur murmurer encore. D'où venait ce murmure; je le cherchai, je le trouvai, il venait de

volonté n'a qu'à vouloir, cette masse obéit à l'instant. Je veux, et tous mes membres se toument comme il me plaît. » RouSSEAu examine lui aussi cette experience, in Émile, liv. IV, p. 574 : « Je veux mouvoir mon bras et je le meus, sans que ce mouvement ait d'autre cause immédiate que ma volonté. C'est en vain qu'on voudrait raisonner pour détruire en moi ce sentiment; il est plus fort que toute évidence; autant vaudrait me prouver que je n'existe pas. » De plus, Rousseau considère avec Fénelon, et selon Descartes, que la matière n'est qu'étendue et figurée, et qu'elle n'est donc pas susceptible de prendre l'initiative du mouvement volontaire : plus généralement, du moment qu'elle est comprise en termes cartésiens, la matière ne peut penser. Au développement que Fénelon consacre à ce problème, dans les Lettres, op. cit. supra n. 21 , lettre I, chap I, $\$ 3$, p. $120:$ «... la raison ne permet pas de croire que la seule matière, quelque figure et quelque mouvement que vous lui donniez, puisse jamais penser, juger, vouloir » - on peut associer le fameux passage de la Profession de foi du vicaire savoyard, in $O C, \mathrm{IV}$, p. 584 : « pour moi je n'ai besoin, quoi qu'en dise Locke, de connaître la matière que comme étendue et divisible pour être assuré qu'elle ne peut penser. » Rappelons au passage que Fénelon envisageait d'une façon moins brutale, avant de l'écarter, l'hypothèse selon laquelle Dieu pourrait joindre à la matière une faculté de sentir et de percevoir - hypothèse que Locke jugeait simplement admissible, mais qu'il ne retenait pas. FÉNELON écrit, en effet, dans les Lettres, op. cit. supra n. 21 , lettre I, chap. I, \$ 4, p. 121 : " Si la pensée n'est qu'un degré d'être que les corps puissent acquérir et perdre, il faut au moins avouer que c'est le plus haut degré d'être que les corps puissent acquérir, et que cette perfection est fort supérieure à celle d'être étendu et figuré. [... Or] la privation d'un degré d'être est le néant de ce degré-là. Pour donner ce degré d'être à celui qui ne l'a point, il faut, pour ainsi dire, travailler sur le néant même, et faire une espèce de création réelle en lui, pour ajouter à l'être inférieur qui existait déjà un nouveau degré d'être qui l'élève au-dessus de lui. » Mais dans ce cas, la création du plus haut degré d'être serait suspendue à l'existence préalable de la matière, ce qui est inadmissible : c'est pourquoi la pensée doit être rapportée à une substance distincte.

44. FÉnelon, op. cit. supra n. 21, lettre II, chap. nI. 
l'amour-propre qui après s'être indigné contre les hommes se soulevait encore contre la raison ${ }^{45}$.

Car l'homme ne peut se résoudre à une nécessité privée de toute justification : l'amour de soi se rebelle contre un niveau de rapports où la personne morale ne trouve pas sa place, et l'amour de l'ordre doit être celui d'un ordre qui, à terme, inclut ma liberté. C'est alors que l'exercice moral de la mémoire rend possible une sorte de reprise à l'égard des étapes de la biographie, dans la mesure où il permet de cerner concrètement le domaine de la volonté droite, qui fonde la liberté authentique. La destinée est la synthèse d'une nécessité aveugle et d'une liberté que la mémoire éprouve en chacun de nous.

André CHARRAK (juin 1996).

45. Huitième promenade, p. 1079. 